\title{
Brain ultrasonography findings in neonates with exposure to cocaine during pregnancy
}

\author{
Marian van Huis • Anne A. M. W. van Kempen • \\ Myrthe Peelen • Maaike Timmers • Kees Boer • \\ Bert J. Smit • Rick R. Van Rijn
}

Received: 28 May 2008 /Revised: 29 October 2008 / Accepted: 8 November 2008 / Published online: 20 December 2008

(C) The Author(s) 2008. This article is published with open access at Springerlink.com

\begin{abstract}
Background Cocaine exposure during pregnancy has been reported to have detrimental effects on the fetus.

Objective To describe the findings on cranial ultrasonography (CUS) as part of a neonatal screening programme for exposed neonates.

Materials and methods The study was a semiprospective analysis of a 12-year cohort of neonates born to mothers who had used cocaine during their pregnancy and who had follow-up according to a strict clinical protocol.

Results In total, 154 neonates (78 boys, 76 girls) were included, of whom 29 (19\%) were born preterm, and 125 (81\%) were born full-term. Abnormalities on CUS were seen in 37 neonates $(24 \%$; $95 \%$ CI $18-31 \%)$. The abnormalities were classified as minor in $20(13 \%$; $95 \%$ CI 9-19\%) and
\end{abstract}

M. van Huis $\cdot K$. Boer

Department of Obstetrics and Gynaecology,

Academic Medical Center Amsterdam,

Amsterdam, The Netherlands

A. A. M. W. van Kempen

Department of Paediatrics, Onze Lieve Vrouwe Gasthuis,

Amsterdam, The Netherlands

M. Peelen $\cdot$ M. Timmers

Faculty of Medicine, Academic Medical Center Amsterdam,

Amsterdam, The Netherlands

\section{B. J. Smit}

Division of Neonatology, Department of Paediatrics,

Erasmus Medical Centre - Sophia Children's Hospital,

Amsterdam, The Netherlands

\section{R. R. Van Rijn $(\bowtie)$}

Department of Radiology, Academic Medical Center Amsterdam,

Meibergdreef 9,

Amsterdam Zuid-Oost 1105 AZ, The Netherlands

e-mail: r.r.vanrijn@amc.uva.nl mildly abnormal in 17 (11\%; 95\% CI 7-17\%). None of the infants showed severe abnormalities. The abnormalities were not associated with the duration or maximum amount of cocaine use during pregnancy.

Conclusion None of the infants had severe abnormalities. Detected abnormalities were not correlated with the duration or maximum amount of cocaine use. Given these findings, we feel that routine cranial ultrasonography in this population is not warranted.

Keywords Neonate $\cdot$ Cocaine $\cdot$ Brain $\cdot$ Ultrasonography

\section{Introduction}

Since the late 1980s intrauterine cocaine exposure has been reported to have detrimental effects on the fetus and to be a risk for pregnancy and delivery $[1,2]$. Currently, cocaine is one of the most popular illicit drugs (besides marijuana) in the Netherlands, being used in all socioeconomic groups. Cocaine has been specifically implicated as a drug with major side effects on pregnancy. In their seminal paper, Dixon and Bejar [3] reported a sevenfold increase in abnormalities on cranial ultrasonography (CUS) in general and a twofold increase in the incidence of subependymal haemorrhage (SEH) in neonates exposed to cocaine and/or methamphetamine. In a relatively small study amongst 21 cocaine users in Amsterdam a 4.3\% neonatal mortality rate was found [4].

Cocaine has two major pharmacological effects. First, it inhibits the reuptake of dopamine, norepinephrine and serotonin. Second, it causes vasoconstriction. As cocaine crosses the placenta, obstetric implications of cocaine use have been reported. These implications are an increased risk of placental abruption [5-7], preterm prelabour rupture 
of membranes [8], and spontaneous abortion. In the neonate, detrimental effects on brain development have been reported $[3,9,10]$. These effects include, amongst others, infarction [11], subependymal cysts [12, 13], haemorrhage, ventricular dilatation, and midline CNS defects [11]. Besides intracranial pathology, congenital malformations, intrauterine growth restriction [14], and neonatal abstinence syndrome [15] have also been reported.

As a result of reports on the effect of cocaine on brain development, the Departments of Obstetrics and Gynaecology, Neonatology and Radiology of the Academic Medical Center Amsterdam instituted screening CUS in neonates with prenatal cocaine exposure. However, more recent reviews of the literature indicated that antenatal cocaine exposure has measurable, but not dramatic effects on infant brain development and CUS studies [16, 17].

The aim of the present semiprospective study was to assess the frequency and severity of abnormalities on CUS, and to assess whether there is an association between the maximum amount or duration of cocaine use during pregnancy.

\section{Materials and methods}

This was a semiprospective study. Consecutive neonates were prospectively included if maternal cocaine use during pregnancy was reported, and CUS was retrospectively analysed. The study was part of a larger semiprospective cohort study of maternal illicit drug users in the greater Amsterdam region. The Department of Obstetrics of the Academic Medical Centre, Amsterdam, led the study. The inclusion period for this retrospective study ran from 1 January 1995 to 31 December 2006.

\section{Participants}

All participants enrolled in this semiprospective study came from a cohort of consecutive neonates born between 1 January 1995 and 31 December 2006 to mothers who used at least cocaine during pregnancy and who were referred to our outpatient obstetric department. Women using cannabis, opiates and methadone, and other illicit drugs besides cocaine were not excluded. Drug use was assessed through extensive drug history, urine screening of the mother, and urine screening of the child. The amount of cocaine use was scored as intermittent (i.e. not daily during pregnancy; usually $<1$ g per occasion), $<1$ g daily, $1-4 \mathrm{~g}$ daily, and $>4 \mathrm{~g}$ daily. Duration of cocaine use was scored as only in the first trimester, in the first and second trimesters, or throughout pregnancy.

If, in any trimester, the mother had a positive history or positive urine test for cocaine or if the neonate had a positive urine test for cocaine, both mother and child were included in the study. In addition, during pregnancy women using cocaine were structurally interviewed by the same midwife (M.v.H.) with respect to quality and quantity of illicit drug use and life-style in each trimester. Clinical data were further obtained through review of medical charts of both mothers and neonates.

In light of the question posed in this study, only those neonates who underwent CUS and whose CUS was considered of sufficient quality for review were analysed.

\section{Cranial ultrasonography}

CUS was performed following the standard routine in place during the study period. Examinations were performed by radiology residents, both with and without supervision of a paediatric radiologist, and paediatric radiologists. CUS was performed using a $7.5-\mathrm{MHz}$ curvilinear transducer and contemporary state-of-the-art equipment. As the studies were performed in a clinical setting there were no specified imaging planes.

CUS studies were retrospectively assessed by one paediatric radiologist (R.v.R.). Although the reviewer was aware of the fact that all studies submitted for review were of neonates with prenatal exposure to cocaine, he was not aware of any clinical findings, maternal nor neonatal, or clinical outcome of the neonates.

The majority of the CUS studies were reviewed on a PACS system (AGFA Impax 4.1 SP4 SU4; Agfa Gevaert, Mortsel, Belgium). A minority of the studies were printed on film and these were of technically adequate quality to review.

Intraventricular haemorrhages were graded according to the procedure of Papile et al. [18]. Increased periventricular echogenicity was scored as either present (periventricular leukomalacia, PVL, grade I) or absent; cystic degeneration was scored as either local (PVL grade II) or disseminated (PVL grade III). Ventricular dilatation was assessed using the Levene index in neonates up to 40 weeks' gestational age (GA) and using the ventricular index in older neonates [19, 20]. The scans were also re-evaluated for the presence of lenticulostriate vasculopathy, calcifications, germinal layer cysts, choroid plexus cysts, and subependymal pseudocysts.

CUS was scored according to procedure of Rademaker et al. [21] and was considered to be normal when no or minor abnormalities, such as germinal layer/plexus cysts, subependymal pseudocysts, or calcifications as exclusive findings were present; mildly abnormal when a haemorrhage grade I or II according to Papile, PVL I, or germinal layer necrosis, or a combination of these features, was present; and severely abnormal when one or more of the following features were present: haemorrhage grade III or IV according to Papile, cystic PVL II/III, thalamic lesion, focal infarction, or convexity haemorrhage. 
Statistical analysis

Statistical analysis was performed using SPSS for Windows 12.0. (Statistical Package for the Social Sciences, Chicago, IL). Results for normally distributed continuous variables (birth weight, GA, etc.) are expressed as means \pm SD. For the neonates, growth data at birth were analysed with the "Kloosterman growth chart" in order to calculate the standard deviation score (SDS) [22].

Bivariate correlations were analysed using a nonparametric test. The following correlations were tested: CUS abnormalities (classified according to Rademaker et al. [21]) versus duration and amount of cocaine use, maternal age, maternal education level, number of prenatal visits, couse of other drugs, alcohol or smoking, complications during pregnancy (intrauterine growth retardation, preeclampsia, HELLP syndrome, or premature labour), complications during delivery (fetal distress, meconium-stained amniotic fluid, premature rupture of membranes, prolonged rupture of membranes, or perinatal infection), GA, birth weight (in grams and expressed as SDS), sex, and 1- and 5min Apgar scores. $P$ values $<0.05$ were assumed to reflect statistical significance.

\section{Ethical approval}

According to Academic Medical Center guidelines for retrospective studies, internal review board approval is not required for the retrospective analysis of clinically obtained data.

\section{Results}

\section{General}

Between 1 January 1995 and 31 December 2006, 188 neonates (97 boys, 52\%; 91 girls, 48\%) were born to 178 women who had used cocaine during pregnancy. Of these women, six had had two pregnancies, one had had three pregnancies, and two delivered twins. The mean age of the mothers at the time of delivery was 29.3 years (SD 6.2 years, range 18.2-42.4 years).

In $15(8 \%)$ of the 188 neonates there was a protocol violation as CUS was not performed. Of the remaining 173, 19 (11\%) CUS studies were of insufficient quality for review and were excluded from further analysis. Of the examinations of insufficient quality for review, 12 were performed by radiology residents (three, $16 \%$, supervised and nine, 47\%, unsupervised), and seven (37\%) were performed by a radiologist. There were no significant differences between neonates with a CUS examination considered to be of sufficient quality for review and those with a CUS examination considered to be of insufficient quality for review with respect to maternal age $(P=0.094)$, GA $(P=0.308)$, birth weight $(P=0.226)$, birth weight SDS $(P=0.735)$, sex distribution $(P=0.264)$, duration $(P=0.298)$, or amount of cocaine use $(P=0.929)$. Review of the radiology reports of the 19 'insufficient quality' examinations revealed no intracranial pathology in 15 neonates (79\%; 95\% CI 56-92\%), in three of which repeat CUS was performed at respectively 1,3 and 5 months after the initial CUS, and these CUS were also reported to be normal. In four neonates $(21 \%$; $95 \%$ CI $8-44 \%)$ abnormalities were seen; in one a choroid plexus cyst and in the other three grade $1 \mathrm{SEH}$.

In total, 154 CUS studies of good quality $(82 \%, 78$ boys and 76 girls, born to 147 mothers) were available for review, and were included in all further analyses.

\section{Maternal characteristics}

In $52(34 \%)$ of the 154 pregnancies, the mother used cocaine in the first trimester only, in $11(7 \%)$ in the first and second trimester only, and in 87 (56\%) also during the third trimester. Two women had a positive urine test in the third trimester only; all other urine tests and histories were negative for cocaine use. Two children had a positive urine test, although the history and/or urine test of the mothers were negative. In $25(16 \%)$ of the pregnancies, the amount of cocaine used by the mother was not known; in $37(24 \%)$ the mother used it intermittently, in $59(38 \%)$ the mother used $<1 \mathrm{~g}$ daily, in $24(16 \%)$ the mother used $1-4 \mathrm{~g}$ daily, and in $9(6 \%)$ the mother used $>4$ g daily.

Co-use of alcohol and nicotine was common in the study population. Alcohol use during pregnancy was recorded in 94 women; 53 (56\%) used alcohol throughout pregnancy. Smoking during pregnancy was recorded in 137 women; 130 (95\%) smoked cigarettes throughout pregnancy. Co-use of other drugs is shown in Table 1.

Table 1 Co-use of other drugs.

\begin{tabular}{ll}
\hline Drugs used & Number (\%) \\
\hline Cocaine only & $20(13.0)$ \\
Cocaine + cannabis & $51(33.1)$ \\
Cocaine + opiates/methadone & $18(11.7)$ \\
Cocaine + other drugs $($ e.g. amphetamines $)$ & $7(4.5)$ \\
Cocaine + cannabis + opiates/methadone & $16(10.4)$ \\
Cocaine + cannabis + other drugs & $19(12.3)$ \\
Cocaine + opiates $/$ methadone + other drugs & $10(6.5)$ \\
Cocaine + cannabis + opiates/methadone + other & $12(7.8)$ \\
$\quad$ drugs & $1(0.6)$ \\
Unknown & \\
\hline
\end{tabular}




\section{Child characteristics}

Nine neonates $(6 \% ; 95 \%$ CI $3-11 \%)$ were born before 33 weeks' GA, 20 (13\%; 95\% CI 8-19\%) between 33 and 37 weeks' GA, and 125 (81\%; 95\% CI 74-87\%) from 37 weeks' GA onwards. The mean birth weight was $2,890 \pm 643 \mathrm{~g}$ (range 780-4,350 g); the mean birth weight SDS was $-0.62 \pm 0.89$ (range -2.72 to +2.18 ). Of the 154 neonates, $36(23 \%$; $95 \%$ CI $17-31 \%)$ were small for GA (birth weight $<-1.3$ SDS), and 4 newborns (3\%; 95\% CI 1$7 \%$ ) were large for GA (birth weight $>+1.3$ SDS). The median Apgar score after 1 min was 9 (range 1-10) and after 5 min was 10 (range 4-10). There were no neonatal deaths in our study cohort (two fetal deaths at 35 and 36 weeks are not included).

Cranial ultrasonography

Cranial ultrasonography was performed by radiology residents in 79 cases (51\%), in 37 cases with supervision, and in 42 cases without supervision; the remaining 75 CUS $(49 \%)$ were performed by radiologists. CUS was performed on average on day 4 (range $0-76$ ). Thirty-seven out of 154 sufficient-quality CUS $(24 \%$; $95 \%$ CI $18-31 \%)$ showed abnormalities on the CUS. Seven of these neonates were born at a GA of $\leq 32$ weeks $(19 \%$; 95\% CI 9-35\%), 8 between 33 and 37 weeks (22\%; 95\% CI $11-37 \%)$ and 22 full term (60\%; $95 \%$ CI $43-74 \%)$.

Table 2 shows the abnormalities on CUS for three GA groups. In total there were 16 grade I SEH in 13 neonates. Of these 13 neonates, 8 (22\%; $95 \%$ CI $11-37 \%)$ showed

Table 2 Distribution of pathology found on routine CUS screening in neonates with prenatal cocaine exposure.

\begin{tabular}{llll}
\hline Abnormality & $\begin{array}{l}\leq 32 \text { weeks } \\
(n=7)\end{array}$ & $\begin{array}{l}33 \text {-37weeks } \\
(n=8)\end{array}$ & $\begin{array}{l}\geq 37 \text { weeks } \\
(n=22)\end{array}$ \\
\hline $\begin{array}{l}\text { Subependymal haemorrhage } \\
\text { Grade 1 }\end{array}$ & 6 & 2 & \\
Grade II & 1 & 0 & 8 \\
Choroid plexus cyst & & & 0 \\
Unilateral & 1 & 4 & 8 \\
Bilateral & 0 & 0 & 0 \\
Subependymal cyst & 1 & 0 & 2 \\
Periventricular echodensity & & 1 \\
Unilateral & 1 & 1 & 1 \\
Bilateral & 1 & 0 & 2 \\
Lenticulostriate vasculopathy & & 5 \\
Unilateral & 0 & 1 & 27 \\
Bilateral & 0 & 0 & 15 \\
Total & 11 & 8 & 7 \\
Rademaker score & & & \\
Minor & 0 & 5 & \\
Mild & 7 & 3 & \\
\hline
\end{tabular}

unilateral grade $1 \mathrm{SEH}$ and $2(5 \%$; $95 \%$ CI 1-19\%) showed bilateral grade $1 \mathrm{SEH}$. One neonate $(3 \%$; $95 \%$ CI $0-15 \%)$ showed a right-sided grade $2 \mathrm{SEH}$ and a left-sided grade 1 SEH. Two neonates (5\%; 95\% CI 1-19\%) showed cystic (old) SEH, one of them bilateral. In 13 out of 37 neonates (35\%; 95\% CI 22-51\%) a choroid plexus cyst was recorded on CUS. Three neonates had a subependymal cyst $(8 \%$; 95\% CI 2-22\%). Five neonates showed increased periventricular echogenicity $(14 \%$; $95 \%$ CI $5-28 \%)$, two of them bilateral. Three neonates had unilateral lenticulostriate vasculopathy $(8 \% ; 95 \%$ CI $2-22 \%)$, and in five it was bilateral (14\%; 95\% CI 5-28\%). One neonate (3\%; 95\% CI $0-15 \%$ ) showed mild bilateral ventricular dilatation without other abnormalities.

Applying the criteria of Rademaker et al. [21], the abnormalities could be classified as minor in $20(13 \%$; 95\% CI 8-19\%) neonates and mildly abnormal in $17(11 \%$; $95 \%$ CI $7-17 \%)$. None of the infants showed severe abnormalities.

Bivariate correlations showed that neither the duration nor the maximum amount of cocaine use during pregnancy was correlated with CUS abnormalities (Table 3). In addition, co-use of other drugs, alcohol use, or cigarette smoking did not correlate with CUS abnormalities. CUS abnormalities were found more frequently with fewer prenatal visits after 20 weeks' GA, in complicated pregnancies, shorter duration of GA, and lower absolute birth weight (Table 3).

Table 3 Correlations with abnormal CUS findings.

\begin{tabular}{|c|c|c|}
\hline & $R$ & $P$ \\
\hline Duration of cocaine use & +0.038 & 0.642 \\
\hline Maximum amount of cocaine use & +0.018 & 0.821 \\
\hline Maternal age & +0.089 & 0.271 \\
\hline Maternal education level & -0.121 & 0.167 \\
\hline Number of prenatal visits before 20 weeks' GA & -0.081 & 0.333 \\
\hline Number of prenatal visits after 20 weeks' GA & -0.220 & 0.006 \\
\hline Co-use of cannabis and/or opiates & -0.101 & 0.213 \\
\hline Alcohol & -0.075 & 0.354 \\
\hline Smoking & -0.048 & 0.557 \\
\hline Complications during pregnancy ${ }^{\mathrm{a}}$ & -0.226 & 0.005 \\
\hline Complications during delivery ${ }^{\mathrm{b}}$ & -0.109 & 0.179 \\
\hline Gestational age (weeks) & -0.488 & $<0.001$ \\
\hline Birth weight $(\mathrm{g})$ & -0.404 & $<0.001$ \\
\hline Birth weight SDS & -0.052 & 0.521 \\
\hline Sex & +0.025 & 0.755 \\
\hline \multicolumn{3}{|l|}{ Apgar scores } \\
\hline $1 \mathrm{~min}$ & -0.039 & 0.640 \\
\hline $5 \mathrm{~min}$ & -0.039 & 0.635 \\
\hline
\end{tabular}

${ }^{\mathrm{a}}$ Intrauterine growth retardation, preeclampsia, HELLP syndrome, or premature labour.

${ }^{\mathrm{b}}$ Fetal distress, meconium-stained amniotic fluid, premature rupture of membranes, prolonged rupture of membranes, or perinatal infection. 


\section{Discussion}

Neonatal congenital anomalies seen on CUS have been related to prenatal cocaine exposure $[13,23,24]$. In our study population $24 \%$ of the neonates with antenatal cocaine exposure showed abnormalities on CUS. Although this seems high, it must be noted that all abnormalities could be classified as minor $(13 \%)$ or mild (11\%); none of the infants had severe CUS abnormalities. In addition, CUS abnormalities were not correlated with the duration or maximum amount of cocaine use.

The literature shows conflicting data with regard to prenatal cocaine exposure in relation to CUS findings. Smith et al. [13] found an increased incidence of subependymal cysts in neonates with prenatal cocaine exposure compared to non-exposed neonates $(44 \%$ vs. $8 \%$, $P<0.01$ ) [13]. Dogra et al. [9] found abnormalities on CUS in 14 of 40 (35\%) neonates, with prenatal cocaine exposure, whereas their age-matched control group showed no abnormalities on CUS. The main findings were focal echolucencies (i.e. cystic lesions) and increased echogenicity of the caudate nucleus.

In a study by King et al. [25], however, no significant difference was found between exposed neonates (incidence $24 \%$ ) and non-exposed neonates (incidence 34\%) with respect to abnormalities found on CUS. These findings are supported by Bauer et al. [15] who compared CUS in 132 exposed neonates to 711 non-exposed neonates. In the exposed group $29.5 \%$ of the neonates and in the nonexposed groups $30.8 \%$ of the neonates showed abnormalities on CUS. As part of the only prospective longitudinal study published to date, 154 cocaine users were matched to 154 control subjects; no significant difference between these groups in the incidence of abnormal CUS (13\% in exposed vs. $8 \%$ in non-exposed neonates) was found [26]. Furthermore, the authors specifically stated that the identified lesions were less severe than previously reported.

There are few data on the influence of the level of cocaine exposure on CUS abnormalities. Only one study assessed a dose-effect relationship between prenatal cocaine exposure and CUS findings [27]. They showed that infants with heavier cocaine exposure were more likely to show SEH than lighter or unexposed infants (adjusted odds ratio 3.88 ; 95\% CI 1.45-10.35). This is in contrast to our results. We performed a subgroup analysis, but this also did not reveal a correlation between SEH and the maximum amount or duration of cocaine use in pregnancy.

An important factor that has received relatively little attention is the relationship between neonatal CUS findings and neurobehavioural outcome in (near-)term infants with antenatal cocaine exposure. Behnke et al. [28] studied 266 infants, both with and without prenatal exposure to cocaine, who underwent CUS within the first $96 \mathrm{~h}$ after birth. There were 239 normal examinations and 27 abnormal examinations. Based on the Amiel-Tison neonatal neurological assessment, performed within $96 \mathrm{~h}$, there was no significant difference between the two groups. Neurodevelopmental outcome has been studied in preterm infants who underwent screening CUS. Rademaker et al. [21] studied a 2-year cohort of 221 preterm infants and related neonatal CUS findings to school performance. Children with normal or mildly abnormal CUS had similar mean IQ values and the proportion of children with an IQ $\leq 85$ was not different between these CUS groups. Pisani et al. [29] followed 78 preterm infants without CUS abnormalities and 50 preterm infants with transient periventricular echodensities up to the age of 12 months [29]. At 12 months, $88.5 \%$ of the first group and $94 \%$ of the second group showed normal neurodevelopment. They concluded that the two groups showed identical outcomes with regard to neurodevelopment. In a study by Stewart et al. [30] of very preterm neonates, 5 of 62 infants (8\%) with normal CUS and 2 of 25 infants (8\%) with uncomplicated periventricular haemorrhage showed neurological or developmental sequelae at 18 months of age.

As in all clinical studies with drug abusers, confounding factors are abundant. In general, socioeconomic status is lower than in the general population. In The Netherlands we generally have a more liberal approach to illicit drug use, which allows early medical support and intervention during pregnancy. This might reduce this effect somewhat. A second confounding factor is the co-use of nicotine, alcohol and other illicit drugs besides cocaine. Teratogenic effects on the developing brain have been described in maternal nicotine and alcohol use. In case of maternal nicotine use, a study in rats has shown that on a cellular level nicotinic cholinergic receptor expression is altered [31]. However, in these cases no gross developmental abnormalities have been described. In prenatal alcohol exposure a reduction in the frontal cortex has been described. This study using CUS as the imaging modality does not allow evaluation of this anomaly [32].

As this was a semiprospective study it had inherent drawbacks. First, US is an operator-dependent technique that makes review a difficult task. Over time, different US systems have been used in our hospital, all reflecting the state-of-the-art at the time of use. As the studies were performed in a clinical setting there were no specified imaging planes for CUS. This led to 19 examinations, containing only a limited number of images, being regarded as inadequate for inclusion in the study. The authors assumed that if at the time of the examination an ultrasonographer had seen an abnormality he/she would likely have stored more images than in an otherwise normal study. Based on this assumption a separate analysis of the US reports in these 19 neonates was performed. This 
showed that exclusion of these cases introduced no bias into our study.

A second drawback is the lack of a control group. We did not attempt to include a control group because we felt it was impossible to compose a group that was completely comparable, especially in life-style and socioeconomic background, to drug users. We therefore had to compare our findings to the findings reported in the literature. Third, it is well known that reliable assessment of drug use is extremely difficult. This obviously has implications for the evaluation of a dose-effect relationship.

Our findings on the occurrence of CUS abnormalities after prenatal cocaine exposure are in keeping with previously published papers $[15,25,26]$. However, it is known that at a later age children with prenatal cocaine exposure may show neurocognitive development disorders. To further study these effects of prenatal cocaine exposure at a later age functional MRI in exposed adolescents, matched to a non-exposed population, would be worth considering.

Although the frequency of CUS abnormalities seems to be high in our study population (around a quarter of the neonates), it corresponds with non-exposed infants in recent reports in the literature. All abnormalities were classified as minor or mild and would probably have no long-term consequences for neurodevelopment. In addition, abnormalities detected with CUS were not correlated with the duration or maximum amount of cocaine use. In spite of the semiprospective nature of our study, we feel on the basis of our findings, in combination with data presented in the literature, that CUS screening in this specific population is not warranted.

Open Access This article is distributed under the terms of the Creative Commons Attribution Noncommercial License, which permits any noncommercial use, distribution, and reproduction in any medium, provided the original author(s) and source are credited.

\section{References}

1. Boer K, Smit BJ, van Huis AM et al (1994) Substance use in pregnancy: do we care? Acta Paediatr Suppl 404:65-71

2. Holzman C, Paneth N (1994) Maternal cocaine use during pregnancy and perinatal outcomes. Epidemiol Rev 16:315-334

3. Dixon SD, Bejar R (1989) Echoencephalographic findings in neonates associated with maternal cocaine and methamphetamine use: incidence and clinical correlations. J Pediatr 115:770-778

4. Smit BJ, Boer K, van Huis AM et al (1994) Cocaine use in pregnancy in Amsterdam. Acta Paediatr Suppl 404:32-35

5. Townsend RR, Laing FC, Jeffrey RB Jr (1988) Placental abruption associated with cocaine abuse. AJR 150:1339-1340

6. Landy HJ, Hinson J (1987) Placental abruption associated with cocaine use: case report. Reprod Toxicol 1:203-205

7. Hulse GK, Milne E, English DR et al (1997) Assessing the relationship between maternal cocaine use and abruptio placentae. Addiction 92:1547-1551
8. Addis A, Moretti ME, Ahmed SF et al (2001) Fetal effects of cocaine: an updated meta-analysis. Reprod Toxicol 15:341369

9. Dogra VS, Shyken JM, Menon PA et al (1994) Neurosonographic abnormalities associated with maternal history of cocaine use in neonates of appropriate size for their gestational age. AJNR 15:697-702

10. Gomez-Anson B, Ramsey RG (1994) Pachygyria in a neonate with prenatal cocaine exposure: MR features. J Comput Assist Tomogr 18:637-639

11. Heier LA, Carpanzano CR, Mast J et al (1991) Maternal cocaine abuse: the spectrum of radiologic abnormalities in the neonatal CNS. AJNR 12:951-956

12. Cohen HL, Sloves JH, Laungani S et al (1994) Neurosonographic findings in full-term infants born to maternal cocaine abusers: visualization of subependymal and periventricular cysts. J Clin Ultrasound 22:327-333

13. Smith LM, Qureshi N, Renslo R et al (2001) Prenatal cocaine exposure and cranial sonographic findings in preterm infants. $\mathrm{J}$ Clin Ultrasound 29:72-77

14. Chazotte C, Youchah J, Freda MC (1995) Cocaine using during pregnancy and low birth weight: the impact of prenatal care and drug treatment. Semin Perinatol 19:293-300

15. Bauer CR, Langer JC, Shankaran S et al (2005) Acute neonatal effects of cocaine exposure during pregnancy. Arch Pediatr Adolesc Med 159:824-834

16. Frank DA, Augustyn M, Zuckerman B (1998) Neonatal neurobehavioral and neuroanatomic correlates of prenatal cocaine exposure. Problems of dose and confounding. Ann N Y Acad Sci 21:40-50

17. Frank DA, Augustyn M, Knight WG et al (2001) Growth, development, and behavior in early childhood following prenatal cocaine exposure: a systematic review. JAMA 285:1613-1625

18. Papile LA, Burnstein J, Burnstein R et al (1978) Incidence and evolution of subependymal and intraventricular hemorrhage: a study of infants with birth weights less than 1,500 gm. J Pediatr 92:529-534

19. Levene MI (1981) Measurement of the growth of the lateral ventricles in preterm infants with real-time ultrasound. Arch Dis Child 56:900-904

20. Shah PS, Sarvaiya JB, Rawal JR et al (1992) Normal ventricular size and ventriculo-hemispheric ratio in infants up to 6 months of age by cranial ultrasonography. Indian Pediatr 29:439-442

21. Rademaker KJ, Uiterwaal CS, Beek FJ et al (2005) Neonatal cranial ultrasound versus MRI and neurodevelopmental outcome at school age in children born preterm. Arch Dis Child Fetal Neonatal Ed 90:F489-F493

22. Kloosterman GJ (1970) On intrauterine growth. Int J Gynaecol Obstet 8:895-912

23. Chasnoff IJ, Bussey ME, Savich R et al (1986) Perinatal cerebral infarction and maternal cocaine use. J Pediatr 108:456-459

24. Haataja L, Mercuri E, Cowan F et al (2000) Cranial ultrasound abnormalities in full term infants in a postnatal ward: outcome at 12 and 18 months. Arch Dis Child Fetal Neonatal Ed 82:F128-F133

25. King TA, Perlman JM, Laptook AR et al (1995) Neurologic manifestations of in utero cocaine exposure in near-term and term infants. Pediatrics 96:259-264

26. Behnke M, Davis EF, Conlon M et al (1998) Incidence and description of structural brain abnormalities in newborns exposed to cocaine. J Pediatr 132:291-294

27. Frank DA, McCarten KM, Robson CD et al (1999) Level of in utero cocaine exposure and neonatal ultrasound findings. Pediatrics 104:1101-1105

28. Behnke M, Eyler FD, Garvan CW et al (1999) Cranial ultrasound abnormalities identified at birth: their relationship to perinatal risk and neurobehavioral outcome. Pediatrics 103:e41 
29. Pisani F, Leali L, Moretti S et al (2006) Transient periventricular echodensities in preterms and neurodevelopmental outcome. J Child Neurol 21:230-235

30. Stewart AL, Thorburn RJ, Hope PL et al (1983) Ultrasound appearance of the brain in very preterm infants and neurodevelopmental outcome at 18 months of age. Arch Dis Child 58:598-604
31. Chen H, Parker SL, Matta SG et al (2005) Gestational nicotine exposure reduces nicotinic cholinergic receptor (nAChR) expression in dopaminergic brain regions of adolescent rats. Eur J Neurosci 22:380-388

32. Wass TS, Persutte WH, Hobbins JC (2001) The impact of prenatal alcohol exposure on frontal cortex development in utero. Am J Obstet Gynecol 185:737-742 